\title{
What makes volcanoes erupt?
}

\author{
Adam J.R. Kent, Christy B. Till, Kari M. COOPER ${ }^{123}$ \\ ${ }^{1}$ Oregon State University, Corvallis OR, 97330, USA, \\ adam.kent@oregonstate.edu \\ ${ }^{2}$ Arizona State Univerity, Tempe, AZ, USA \\ ${ }^{3}$ University of California at Davis, Davis, CA, USA.
}

One key aspect of volcanic eruptions are the mechanisms by which magmas go from stable storage within the crust, to ascent and eventual eruption; described herein as eruption initiation. Although this process is often considered simplistically, a detailed understanding of the nature and timing of the physical and chemical processes that lead to eruption initiation is an important consideration for resurrecting the past behavior of volcanic systems from the geological record, for interpreting volcano monitoring data, and for considering hazards and responses associated with future eruptions. Petrological approaches are particularly amenable for studying eruption initiation, as they typically involve some of the last high temperature processes that affect erupted magmas, and thus often leave clear signals preserved in the petrographic record. In addition petrological and related techniques can provide the means to determine the timescale associated with eruption initiation.

A global survey of eruption initiation mechanisms deduced from petrology and other observations shows that the most common initiation processes preserved in the petrologic record are mafic recharge, where resident silicic magmas are reactivated by addition of more mafic magma, and rejuvenation, where hotter magmas of broadly similar bulk composition are added. For rejuvenation we can also identify both mafic rejuvenation and felsic rejuvenation as this process occurs in both mafic and silicic systems. Increases in overpressure associated with vapor accumulation may also leave a petrologic record, but are less commonly documented. Other proposed mechanisms of eruption initiation such as critical buoyancy, roof failure, or far field triggering, are less likely to leave unambiguous petrologic signatures.

We have documented the erupted volume, composition, style and eruption timescale (the time between initiatiom iniotiation in the subsrface and eventual eruption) for over 70 eruptions at 40 volcanoes worldwide Our results show that statistically significant differences in all these parameters are associated with different eruption mechanisms. This in turn suggests that prior knowledge of the likely eruption initiation mechanism, obtained via moonitoring observations and/or study of previous eruptions, has important predictive power. 